\title{
Looking for gravitational errors
}

\section{The idea that there may be forces between macroscopic objects other than those caused by gravity is no} longer an outrage. But precisely what is meant remains unclear.

THERE is always a special attraction in the discovery in the published literature of a surprising truth that other people have overlooked. This is no doubt one of the extra reasons why close attention was paid to the announcement earlier this year, by Fischbach et al. that the Eotvos experiments, first published in the 1920 s and widely regarded since then as a proof that the gravitational force between massive objects is independent of their chemical constitution, concealed evidence that there is a force other than gravity between ordinary material objects (see Nature 319, $173 ; 1986)$. The proposal is not nearly as heterodox as it may at first have seemed. Most obviously, there have repeatedly been suggestions that the constant in Newton's law of gravitation (called $G$ ) is smaller when measured in laboratoryscale experiments than the value required to account for geophysical phenomena.

The article by Fischbach et al. originally appeared in Phys. Rev. Lett. $(\mathbf{5 6}, 3 ; 1986)$. The essence of its conclusions was that the published errors in the Eotvos measurements of the gravitational attraction between different kinds of substances (carried out with a torsion balance) could be accounted for if there is a force with a characteristic range of about $200 \mathrm{~m}$ whose strength depends on the baryon number (only roughly proportional to the mass) of the interacting materials. Now the same journal has published three papers which take the argument a little further, but which have the virtue of suggesting how the matter might be settled.

It is not really surprising that effects such as that described by Fischbach et al., if real, should have been overlooked for half a century. In spite of the exquisite accuracy with which it is possible to describe gravitational systems such as planets in orbit about a central mass such as the Sun, these successes cannot exclude the possibility that short-range forces between material objects may exist. Moreover, in systems such as these, where only relative masses matter to a first approximation, uncertainties may be arbitarily accommodated in either $G$ or in the planetary and solar masses. This is one reason why it is notoriously a minor scandal that $G$ is, even now, known a little less accurately than one part in $10^{5}$

For the sake of clarity, it is worth recalling the form in which the Fischbach result is put. Most simply, $G$ can be set equal to $G_{i n}$, the value of the gravitational constant at infinity, multiplied by a factor differing from one by the addition of a relatively small distance-dependent exponential term of the form $\exp ^{-f r l)}$. The distance $l$ then represents the scale over which departures from strict compliance with Newton's inverse-square law may be expected. The supposed constant by which the exponential factor is multiplied is a measure of the strength of the non-newtonian effect. The characteristic length was estimated at $200 \mathrm{~m}$, with a possible error of 50 $\mathrm{m}$ in either direction. The constant of proportionality is most distinctive for being negative, meaning that apparently gravitational forces are smaller at smaller distances, or that the extra force is repulsive; its magnitude is such as to affect gravitational attraction by a few tenths of a per cent at zero distance.

It is also, of course, possible that some of the uncertainty in the value of $G$ arises because of attempts to reconcile irreconcilable measurements of gravitational forces on the very large scale and the very small. If, indeed, there is an extra force of interaction perceptible over distances of a few hundred metres only, laboratory measurements of gravitation, going back to Cavendish, will differ systematically from those inferred from astronomical observations. Certainly one of the consequences of the reinterpretation of the Eotvos experiments by Fischbach et al. is that future attempts to narrow the errors in measurements of gravitational force will diligently segregate short-range from long-range measurements.

Two of the clutch of three articles now published by Physical Review Letters are, like Fischbach et al., of an archival character. Both David A. Neufeld of Harvard University $(56,2344 ; 1986)$ and S. Nussinor of Tel Aviv University (56, 2350; 1986) go back to the records of an experiment by L.B. Kreuzer first published in 1968 (Phys. Rev. 169, 1007; 1968). The objective was to carry out a kind of dynamical equivalent of Cavendish's direct measurement of the gravitational force between two static objects. For this purpose, Kreuzer used as gravitating masses containers with equal volumes of liquid within which were embedded oscillating masses of density equal to that of the surrounding fluid (which is a neat way of ensuring that the centres of gravity of the two objects do not change with the oscillation). The oscillation itself was intended to provide a signal of known frequency that, with luck and clever design, might be picked out easily from among the inevitable noise. Kreuzer's objective was to distinguish between the "passive" and "active" or dynamic gravitational fields caused by moving masses; his conclusion was that they could differ by, at most, one part in 20,000 .

Both Neufeld and Nussinov now point out that the same measurements can be interpreted in terms relevant to the new Fischbach force. For, by good luck, the fluid in the Kreuzer experiment was a mixture of halogen-containing hydrocarbons different in chemical constitution from the solid rods of Teflon suspended in them. The difference is enough to account for a significant difference, for each unit mass, in baryon number, which is a measure of the content of protons and neutrons in a material (whereas the mass of the same material is the same quantity modified by what the nuclear physicists call the packing fraction, or the nuclear binding energy, of the elementary constituents of the materials).

Both Neufeld and Nussinov now argue that the Kreuzer measurements probably provide a more sensitive test of the implications drawn from the Fischbach analysis than an attempt to repeat the Eotvos experiments as such. Indeed, on various interpretations of the Kreuzer measurements, the intermediate-range field of force suggested by the re-analysis is already a little beyond the bounds of likelihood. Neufeld suggests that if the Eotvos measurements are to be repeated, it would be most profitable to do so at the foot of a tall cliff, where the effects of natural gravitational asymmetry would be most apparent.

P. Thieberger from the Brookhaven National Laboratory $(\mathbf{5 6}, \mathbf{2 3 4 7} ; \mathbf{1 9 8 6})$ is even more lyrical on the subject: repeating the Eotvos measurements at the bottom and the top of a tall cliff could be the most sensitive measurements yet bearing on the existence (or otherwise) of the intermediate force. (The University of Bath, sited on the top of such a cliff, is well placed, among others.) Given this enthusiasm it is certain that there will now be a host of proposals for the re-measurement of gravity by classical devices. The driving force may be the prize of pinning down the intermediate force. It must be hoped that one by-product will be the even more important definition of an accurate value of $G$.
John Maddox 